\title{
The Performance of CURB-65 and PSI for Predicting In-Hospital Mortality of Community-Acquired Pneumonia in Patients with Type 2 Diabetes Compared with the Non-Diabetic Population
}

This article was published in the following Dove Press journal:

Diabetes, Metabolic Syndrome and Obesity: Targets and Therapy

\section{Chun-Ming $\mathrm{Ma}^{\prime}$ \\ Ning Wang ${ }^{2}$ \\ Quan-Wei Su ${ }^{3}$ \\ Ying Yan $^{3}$ \\ Fu-Zai Yin (D)'}

'Department of Endocrinology, The First Hospital of Qinhuangdao, Qinhuangdao, Hebei, People's Republic of China; ${ }^{2}$ Department of Internal Medicine, Hebei Medical University, Shijiazhuang, Hebei, People's Republic of China; ${ }^{3}$ Department of Internal Medicine, Chengde Medical College, Chengde, Hebei, People's Republic of China
Correspondence: Fu-Zai Yin Email yinfuzai62@163.com

\begin{abstract}
Objective: To compare the performance of CURB-65 and Pneumonia Severity Index (PSI) for predicting in-hospital mortality of community-acquired pneumonia (CAP) between patients with and without type 2 diabetes (T2DM).

Methods: A retrospective study was conducted on 2365 CAP patients in The First Hospital of Qinhuangdao, China. The primary outcome was in-hospital mortality. The area under curves (AUCs) was used to evaluate the abilities of CRB-65, CURB-65, and PSI class for predicting in-hospital mortality in patients with CAP.

Results: Among CAP patients, 127 patients (5.4\%) died, 80 patients were without diabetes, and 47 patients had T2DM. In-hospital mortality increased with the risk stratification defined as CURB-65 and PSI class in both non-diabetes and T2DM patients $(P<0.05)$. The AUCs for predicting in-hospital mortality were $0.728 \sim 0.798$ in patients without T2DM (CRB-65: 0.728, CURB-65: 0.757 and PSI class: 0.798 ) and $0.641 \sim 0.716$ in patients with T2DM (CRB-65: 0.641, CURB-65: 0.677 and PSI class: 0.716)( $P<0.001)$. The AUC of the PSI class was lower in patients with T2DM than in patients without T2DM $(P<0.05)$.

Conclusion: CURB-65 and PSI class are correlated with in-hospital mortality of CAP in patients with and without T2DM. Compared with non-diabetes patients, the predictive performance of CURB-65 and PSI class decreased in patients with T2DM. A prediction model for evaluating the CAP severity in the T2DM population should be developed by future studies.
\end{abstract}

Keywords: community-acquired pneumonia, CURB-65, pneumonia severity index, mortality, type 2 diabetes

\section{Introduction}

Community-acquired pneumonia (CAP) is a very common respiratory disease and an important cause of mortality and morbidity worldwide. The incidence of CAP ranges from 1.76 to 9.6 per 1000 person-years. ${ }^{1-5}$ CAP patients may have different outcomes varying from rapid recovery to death. Therefore, it is crucial to evaluate the severity of CAP before starting treatment. CURB-65 and Pneumonia Severity Index (PSI) are two commonly used tools for evaluating the severity of CAP, ${ }^{6,7}$ which have been recommended by several CAP guidelines. ${ }^{8-11}$

Diabetes is another major global health problem. According to the latest data from International Diabetes Federation, there were 463 million adults living with 
diabetes in 2019. Type 2 diabetes, which is the most common type of diabetes, ${ }^{12}$ can cause a series of microvascular and macrovascular complications to vital organs, including the lungs. ${ }^{13}$ Diabetes can destroy immune response, affect airway glucose homeostasis, thus reducing the pulmonary function. ${ }^{15-17}$ Pneumonia is considered a pulmonary complication of diabetes. ${ }^{14}$ Studies have suggested that diabetes can increase hospitalization risk in patients with CAP, thus leading to higher mortality and increased cost of treatment. ${ }^{18-21}$

It remains unclear whether CURB-65 and PSI can be used to evaluate the severity of CAP in patients with diabetes. The aim of this study was to compare the performance of CURB-65 and PSI for predicting in-hospital mortality of community-acquired pneumonia between patients with and without type 2 diabetes in First Hospital of Qinhuangdao, China.

\section{Methods}

\section{Subjects}

This retrospective study included patients hospitalized at the First Hospital of Qinhuangdao between January 2015 and December 2018. The inclusion criteria were the following: 1) all patients were diagnosed with CAP, 2) patients over 18 years of age. The exclusion criteria were: 1) patients with type 1 diabetes; 2) patients with other specific types of diabetes; 3) patients with no clear type classification; 4) patients with pre-diabetes; 5) pregnancy; 6) obstetric infection; 7) clinical data about CURB65 and PSI missing. This study was approved by the ethics committee of the First Hospital of Qinhuangdao.

\section{Definition of CAP and Classification of Diabetic Type}

The definition of CAP was done according to the 2016 CAP clinical practice guidelines by the Chinese Thoracic Society. ${ }^{8}$

Diabetic types were classified using the International classification of Diseases-10 (ICD-10). The codes of T2DM were included in the study. The codes of type 1 diabetes and other specific types of diabetes were excluded. Nonspecific codes and codes of pre-diabetes were also excluded in the study.

\section{Data Collection}

Initial data after admission were extracted from the Hospital Information System. Sociodemographic variables included age, sex, and ethnicity. Clinical data included the diagnosis and classification of diabetes, neoplastic disease, liver disease, congestive heart failure, cerebrovascular disease, and renal disease. Physical examination included mental status, respiratory rate, blood pressure, temperature, and pulse. Laboratory data included arterial $\mathrm{pH}$, $\mathrm{PaO}_{2}, \mathrm{SaO}_{2}$, urea, sodium, glucose, and hematocrit. Pleural effusion was also collected. The scores of CRB65 [confusion, respiratory rate $\geq 30 / \mathrm{min}$, blood pressure (systolic blood pressure $<90 \mathrm{mmHg}$ or diastolic blood pressure $\leq 60 \mathrm{mmHg}$ ) and age $\geq 65$ years], CURB-65 [CRB65 plus urea $>7 \mathrm{mmol} / \mathrm{L}$ ] and PSI were calculated. ${ }^{6,7}$ Patients with a CRB-65 score 0 were defined as a lowrisk group, with a score $1 \sim 2$ as an intermediate-risk group, and with a score $\geq 3$ as a high-risk group. Patients with a CURB-65 score $0 \sim 1$ were defined as a low-risk group, with score 2 as an intermediate-risk group, and with scores $3 \sim 5$ as a high-risk group. According to the PSI score, patients were classified into five risk classes. Patients with PSI class I $\sim$ III were defined as a low-risk group, with class IV as an intermediate-risk group, and with class $\mathrm{V}$ as a high-risk group. The primary outcome was in-hospital mortality.

\section{Statistical Analyses}

All analyses were performed using the SPSS 24.0 statistical software (SPSS 24.0 for Windows; SPSS, Inc., Chicago, IL). Numerical variables were not normally distributed and were expressed as medians (interquartile range). Comparisons were conducted between groups using the Mann-Whitney $U$-test. Categorical data were reported as abnormal subjects (\%) and were analyzed using the chi-square test or Fisher's exact test. The relationship between the risk stratification and in-hospital mortality was analyzed by multiple logistic regression, adjusted for sex and age. The area under curves (AUCs) for CRB-65, CURB-65, and PSI class were drawn by receiver operating characteristic curve (ROC curve) analysis. AUCs were used to evaluate the abilities of CRB-65, CURB-65, and PSI classes for predicting in-hospital mortality in patients with CAP. The performance of CRB-65, CURB-65, and PSI for predicting in-hospital mortality of CAP was compared between patients with and without T2DM. The ROC curve analysis was performed with MedCalc15.2.2 software (Ostend, Belgium). $P<0.05$ was considered statistically significant. 


\section{Results}

A total of 2682 CAP patients were analyzed. Thirty-six patients with type 1 diabetes, other specific types of diabetes, or no clear type classification, and 23 patients with pre-diabetes were excluded from the study, as well as 8 patients with pregnancy or obstetric infection. Two hundred fifty patients were additionally excluded due to missing clinical data (CURB-65 and PSI). Eventually, this study enrolled 2365 patients (1372 males and 993 females), aged 66.6 \pm 17.3 years (Figure S1). Among those patients, 531 patients (22.5\%) had T2DM.

Among the CAP patients, 127 patients (5.4\%) died, 80 patients were without diabetes, and 47 patients had T2DM. The percentage of chronic obstructive pulmonary disease (COPD) and asthma were similar between those patients with and without T2DM $(P>0.05)$ (Table S1). The scores of CRB-65, CURB-65, and PSI were all significantly higher in T2DM patients than non-diabetes patients $(P<0.001) \quad$ (Table S2). In-hospital mortality increased with the risk stratification defined as CRB-65, CURB-65, and PSI class $(P<0.05)$. Moreover, in-hospital mortality increased with the risk stratification defined as CURB-65 and PSI class in both non-diabetes and T2DM patients $(P<0.05)$ (Figure 1, Table S3).

The characteristics of patients with different clinical outcomes are shown in Table 1. The scores of CRB-65, CURB-65, and PSI were significantly higher in death patients than in those who survived $(P<0.001)$. In nondiabetic and T2DM patients, the scores of CRB-65, CURB-65, and PSI were all significantly higher in death patients than survival patients $(P<0.001)$. In survival patients, the scores of CRB-65, CURB-65, and PSI were all significantly higher in T2DM patients than in nondiabetic patients $(P<0.001)$. In death patients, the scores of CRB-65, CURB-65, and PSI were similar between T2DM patients and non-diabetic patients $(P>0.05)$ (Table 1). The number (\%) of patients with each CRB-65 score, CURB-65 score, and PSI class are presented in Table S4.

The AUCs for predicting in-hospital mortality were $0.705 \sim 0.782(P<0.001)$ (Figure 2, Table S5). The AUC of CURB-65 was higher than the AUC of CRB-65 $(P<0.05)$. The AUC of the PSI class was higher than the AUCs of CRB-65 and CURB-65 $(P<0.05)$. In patients with T2DM, the AUC of the PSI class was higher than the AUC of CRB-65 $(P<0.05)$. The AUCs for predicting in-hospital mortality were $0.728 \sim 0.798$ in patients without $\mathrm{T} 2 \mathrm{DM}$ and $0.641 \sim 0.716$ in patients with $\mathrm{T} 2 \mathrm{DM}$
$(P<0.001)$. The AUC of the PSI class was lower in patients with T2DM than in patients without T2DM $(P<0.05)$ (Figure 2, Table S5). For both non-diabetic and T2DM patients the optimal cut-off points were CRB-65 score 2, CURB-65 score 2, and PSI class IV. The sensitivities, specificities, and Youden's index are shown in Table 2. For non-diabetic patients, the sensitivity of CRB-65 was $45.0 \%$ and the specificity of CRB- 65 was $86.1 \%$; the sensitivity of CURB- 65 was $70.0 \%$ and the specificity of CURB-65 was $70.2 \%$; the sensitivity of PSI was $83.8 \%$ and the specificity of PSI was $68.0 \%$. For T2DM patients, the sensitivity of CRB- 65 was $40.4 \%$ and the specificity of CRB-65 was $83.1 \%$; the sensitivity of CURB-65 was $72.3 \%$ and the specificity of CURB-65 was $59.5 \%$; the sensitivity of PSI was $83.0 \%$ and the specificity of PSI was $49.0 \%$.

\section{Discussion}

Our study shows that in-hospital mortality increased with the CAP risk stratification in both non-diabetes and T2DM patients. Nevertheless, the AUCs for predicting in-hospital mortality were lower in T2DM patients compared with non-diabetic patients. When the sensitivities were similar, the specificities were lower in T2DM patients than nondiabetic patients.

In diabetes stratified analyses, the AUCs were $0.728 \sim$ 0.798 in the non-diabetic group and $0.641 \sim 0.716$ in the T2DM group. The AUCs dropped by $0.080 \sim 0.087$ in patients with T2DM compared to patients without T2DM. However, the difference was statistically significant only in PSI. AUC is an index for evaluating the discrimination of the prediction model. The reduction of AUC reflects poor discrimination in T2DM. In the predictive model of death, we expected death patients to have a high-risk score and survival patients to have a low-risk score. In our study, the scores of CRB-65, CURB-65, and PSI were all high in death patients with or without T2DM. However, the scores of CRB-65, CURB-65, and PSI were higher in survival patients with T2DM than those without T2DM. Hence, the difference of CRB-65, CURB-65, and PSI between survival and death patients shrunk in T2DM. The power of discrimination decreased. Due to the similar score in death patients, the sensitivities were similar. The specificities were low in T2DM because of the higher scores in survival patients with T2DM.

The development of CURB-65 was based on three large prospective studies of CAP from the United Kingdom, New Zealand, and the Netherlands. The 

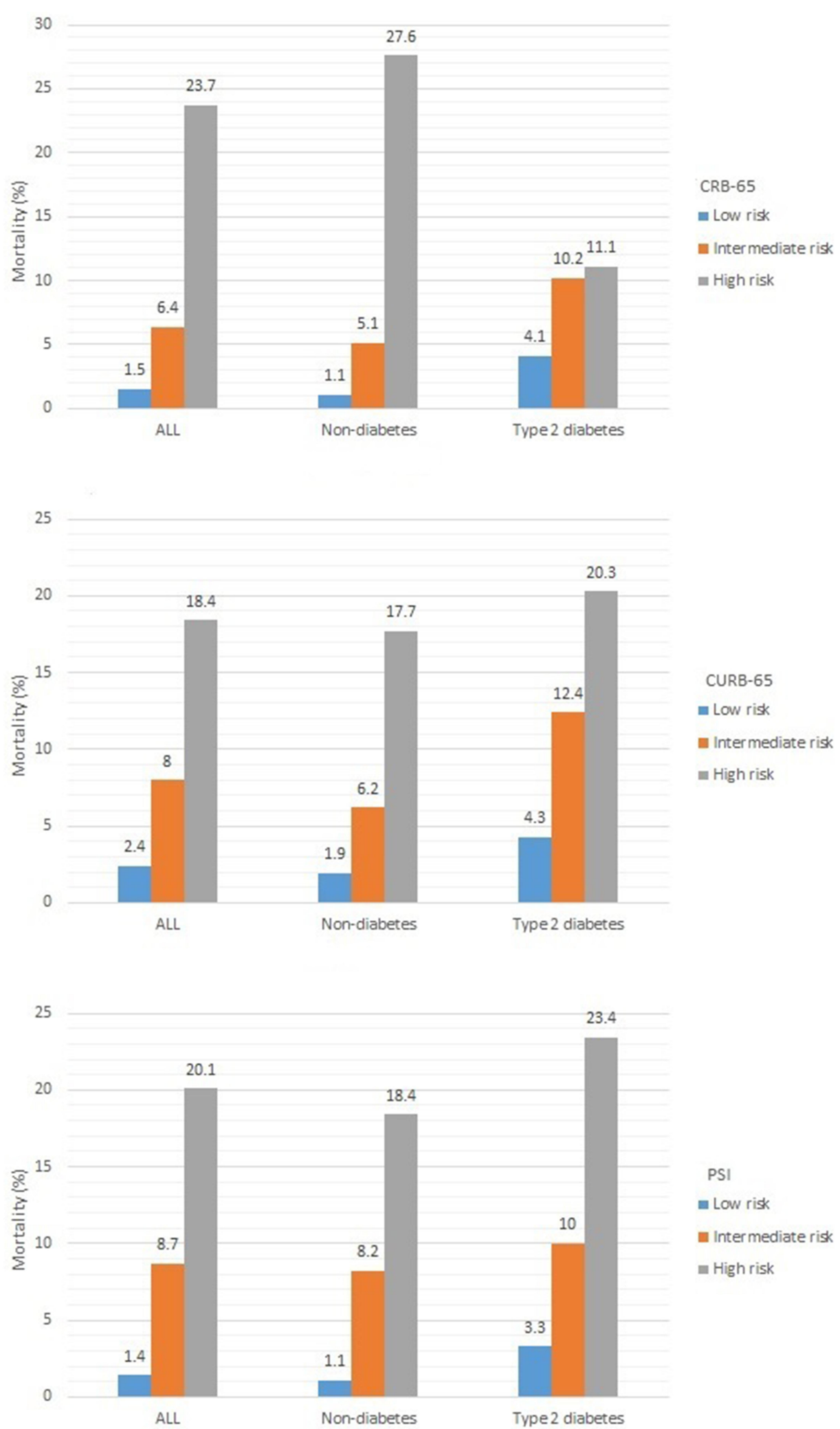

Figure I The mortality in community-acquired pneumonia patients with different risk stratification.

development of PSI was based on MedisGroups European and American populations, the accuracy of Comparative Hospital Database from the United States. CURB-65 and PSI have been validated in an Asian popuAlthough CURB-65 and PSI were developed in the lation. The AUCs of CURB-65 and PSI in predicting 


\begin{tabular}{|c|c|c|c|c|c|c|}
\hline \multirow{3}{*}{ 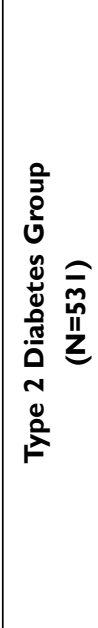 } & 0 & & 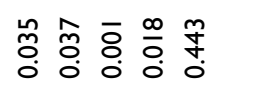 & $\overline{\mathrm{o}} \overline{\mathrm{i}}$ & &  \\
\hline & 㔛 & & 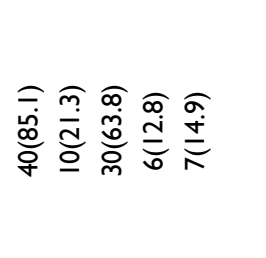 & 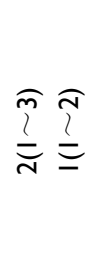 & & 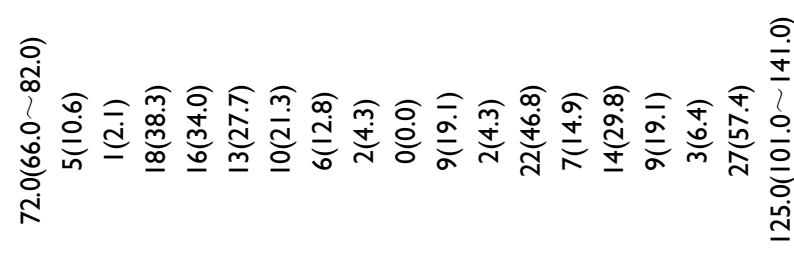 \\
\hline & 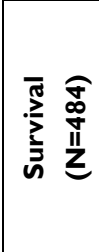 & & 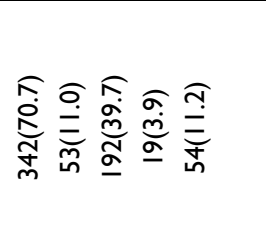 & $\begin{array}{l}\stackrel{\leftrightarrow}{i} \stackrel{\leftrightarrow}{=} \\
\stackrel{?}{=}\end{array}$ & & 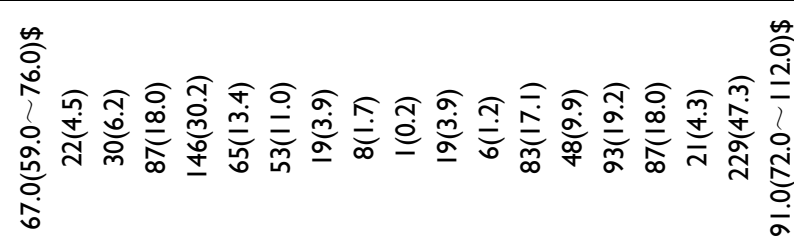 \\
\hline \multirow{3}{*}{ 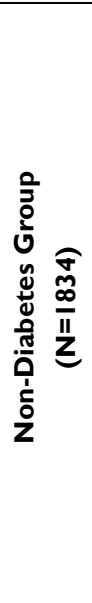 } & 0 & & 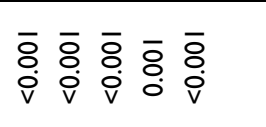 & $\overline{8} \overline{\dot{o}}$ & & 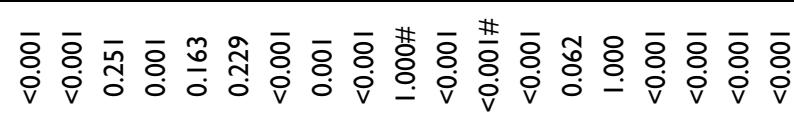 \\
\hline & 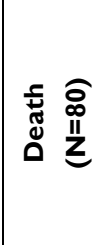 & & 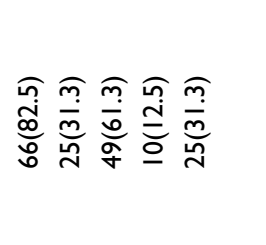 & 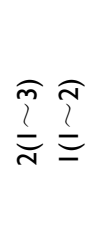 & & 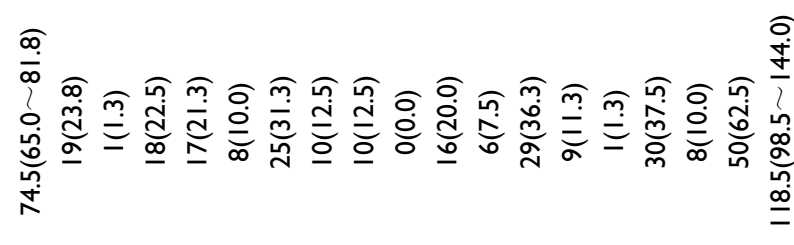 \\
\hline &  & & 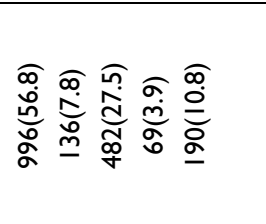 & 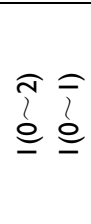 & & 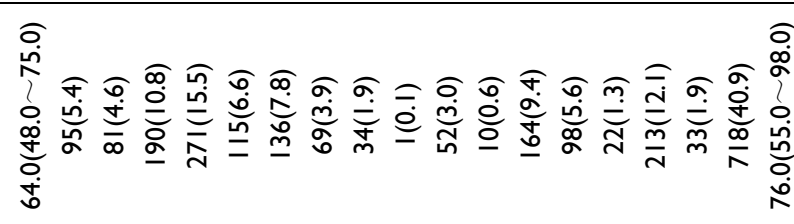 \\
\hline \multirow{3}{*}{ 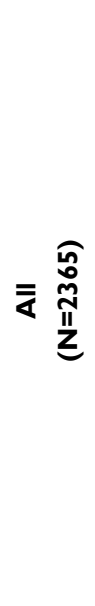 } & 0 & & 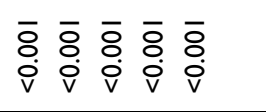 & $\begin{array}{l}\overline{8} \overline{0} \\
\dot{0} \dot{0} \\
\mathrm{v}\end{array}$ & & 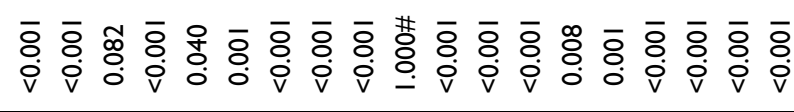 \\
\hline & 吾 $\frac{\widehat{I}}{\underline{I}}$ & & 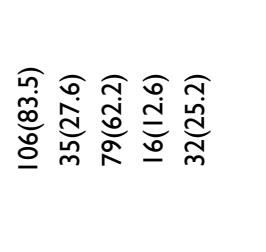 & 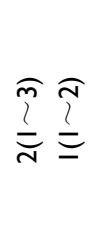 & & 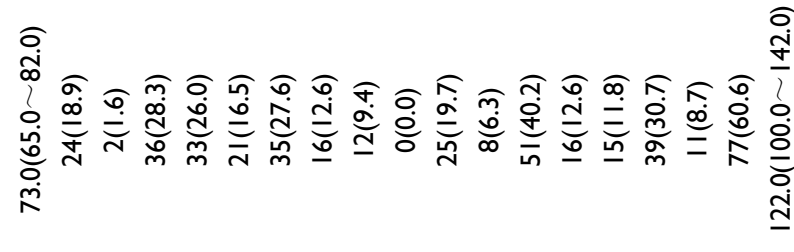 \\
\hline & 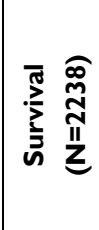 & & 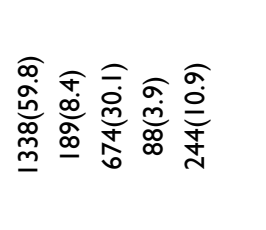 & $\begin{array}{l}\widehat{\hat{\imath}} \\
\hat{\imath} \\
\underline{0}\end{array}$ & & 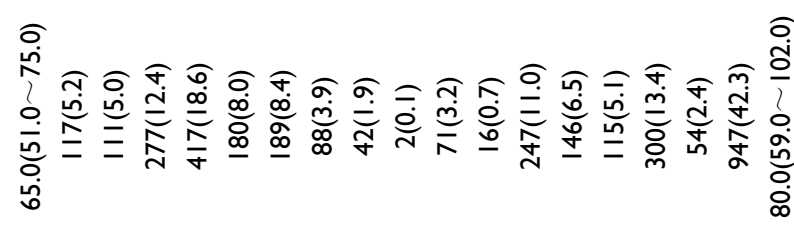 \\
\hline$\frac{\frac{\pi}{0}}{\frac{\pi}{\frac{\pi}{\pi}}}$ & & & 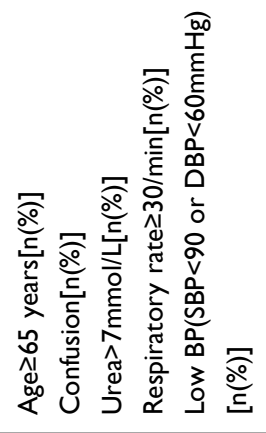 &  & 0 &  \\
\hline
\end{tabular}



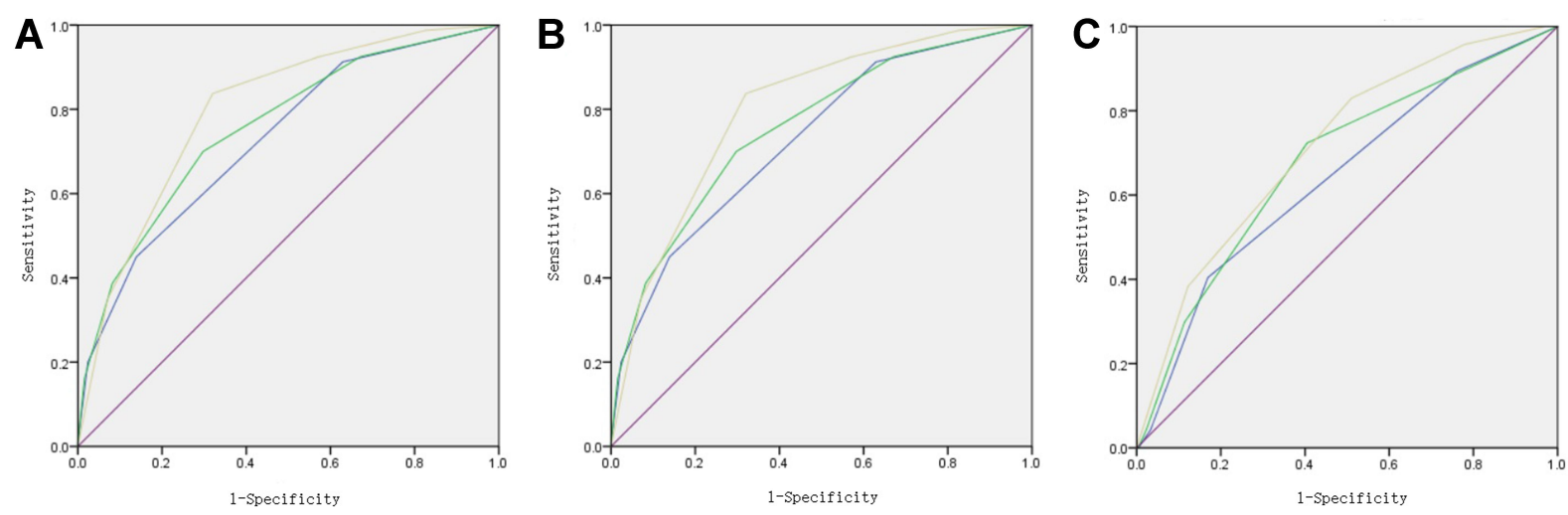

Figure 2 The receiver operator characteristic curve of CURB-65 and PSI for evaluating the risk of death in community-acquired pneumonia inpatients. (A) All patients; (B) non-diabetic patients; (C) type 2 diabetic patients.

Abbreviation: PSI, pneumonia severity index.

28-day mortality were 0.829 and 0.813 in Beijing Chaoyang Hospital; ${ }^{22}$ the AUCs of CRB-65, CURB-65, and PSI in predicting 30-day mortality were $0.694 \sim 0.736$ in Hong Kong; ${ }^{23}$ the AUCs of CURB-65 and PSI in predicting 28-day mortality were 0.701 and 0.735 in Korea; ${ }^{24}$ the AUCs of CURB-65 and PSI in predicting 30-day mortality were 0.755 and 0.767 in Japan. ${ }^{25}$ In summary, the accuracies of CURB-65 and PSI were moderate in the Asian populations. ${ }^{26}$ Similar to the above study, we found that the AUCs of CRB-65, CURB-65, and PSI in predicting in-hospital mortality were $0.705 \sim 0.782$.

The Clinical Practice Guideline of the American Thoracic Society and Infectious Diseases Society of America recommends using the PSI over the CURB-65

Table 2 The Sensitivities, Specificities and Youden's Index of CURB-65 and PSI for Evaluating the Risk of Death in CommunityAcquired Pneumonia Inpatients

\begin{tabular}{|c|c|c|c|c|c|c|c|c|c|c|}
\hline \multicolumn{2}{|l|}{ Variables } & \multicolumn{3}{|c|}{$\begin{array}{c}\text { All } \\
(\mathrm{N}=2365)\end{array}$} & \multicolumn{3}{|c|}{$\begin{array}{l}\text { Non-Diabetes Group } \\
(\mathrm{N}=1 \mathbf{8 3 4})\end{array}$} & \multicolumn{3}{|c|}{$\begin{array}{c}\text { Type } 2 \text { Diabetes Group } \\
(\mathrm{N}=53 \mathrm{I})\end{array}$} \\
\hline CRB-65 & Score & Sen(\%) & Spe(\%) & $\begin{array}{l}\text { Youden's } \\
\text { Index }\end{array}$ & Sen(\%) & Spe(\%) & $\begin{array}{l}\text { Youden's } \\
\text { Index }\end{array}$ & Sen(\%) & Spe(\%) & $\begin{array}{l}\text { Youden's } \\
\text { Index }\end{array}$ \\
\hline & 0 & 100 & 0 & 0.000 & 100 & 0 & 0.000 & 100 & 0 & 0.000 \\
\hline & I & 90.6 & 34.2 & 0.248 & 91.3 & 37.1 & 0.283 & 89.4 & 24.0 & 0.133 \\
\hline & 2 & 43.3 & 85.4 & 0.287 & 45.0 & 86.1 & 0.311 & 40.4 & 83.1 & 0.235 \\
\hline & 3 & 14.2 & 97.4 & 0.116 & 20.0 & 97.6 & 0.176 & 4.3 & 96.7 & 0.009 \\
\hline & 4 & 0.8 & 99.9 & 0.007 & 1.3 & 99.9 & 0.012 & 0 & 99.6 & -0.004 \\
\hline \multirow[t]{7}{*}{ CURB-65 } & Score & & & & & & & & & \\
\hline & 0 & 100 & 0 & 0.000 & 100 & 0 & 0.000 & 100 & 0 & 0.000 \\
\hline & I & 92.1 & 29.7 & 0.218 & 92.5 & 32.8 & 0.253 & 91.5 & 18.2 & 0.097 \\
\hline & 2 & 70.9 & 67.9 & 0.388 & 70.0 & 70.2 & 0.402 & 72.3 & 59.5 & 0.318 \\
\hline & 3 & 35.4 & 91.1 & 0.265 & 38.8 & 91.8 & 0.305 & 29.8 & 88.6 & 0.184 \\
\hline & 4 & 11.8 & 98.3 & 0.101 & 16.3 & 98.4 & 0.147 & 4.3 & 97.7 & 0.020 \\
\hline & 5 & 0.8 & 99.9 & 0.007 & 1.3 & 99.9 & 0.012 & 0 & 99.6 & -0.004 \\
\hline \multirow[t]{6}{*}{ PSI } & Class & & & & & & & & & \\
\hline & I & 100 & 0 & 0.000 & 100 & 0 & 0.000 & 100 & 0 & 0.000 \\
\hline & II & 99.2 & 14.2 & 0.134 & 98.8 & 17.3 & 0.161 & 100 & 2.9 & 0.029 \\
\hline & III & 93.7 & 38.2 & 0.319 & 92.5 & 42.6 & 0.351 & 95.7 & 22.1 & 0.179 \\
\hline & IV & 83.5 & 63.9 & 0.473 & 83.8 & 68.0 & 0.517 & 83.0 & 49.0 & 0.319 \\
\hline & V & 36.2 & 91.8 & 0.280 & 35.0 & 92.9 & 0.279 & 38.3 & 87.8 & 0.261 \\
\hline
\end{tabular}

Abbreviations: PSI, pneumonia severity index; Sen, sensitivity; Spe, specificity. 
to determine the need for hospitalization in CAP. ${ }^{9}$ Aujesky et al found that PSI has higher discriminatory power for short-term mortality than CURB-65. ${ }^{27}$ Moreover, Zhang et al found that PSI performed significantly better than CURB-65 for mortality prediction. ${ }^{28}$ The meta-analysis also found that PSI had the highest sensitivity for mortality. ${ }^{29}$ In our study, the performance of PSI was also superior to CRB-65 and CURB-65. The AUC and sensitivity of PSI were higher than CRB-65 and CURB65. The specificity of PSI was similar to CURB-65. Nonetheless, PSI includes many variables, which complicates its implementation.

Aging is associated with high mortality in CAP patients. ${ }^{30}$ The discriminative power of CURB-65 and PSI decreases with aging. ${ }^{28}$ In our study, the age was generally high in T2DM patients, even in survival patients, which may be the main reason why the scores were higher in survival T2DM patients. Another reason could be associated with concomitant disease. T2DM often coexists with multiple concomitant diseases, such as chronic kidney disease, heart failure, cerebrovascular diseases, and so on. ${ }^{31-33}$ In our study, these concomitant diseases were also common in survival T2DM patients.

This study has a few limitations. First, the predictive accuracy of CURB-65 and PSI was poor in influenza pneumonia. ${ }^{34}$ The constituents of etiology may affect the performances of CURB-65 and PSI. However, it was not possible to obtain the information on the aetiology of CAP. Second, the nursing home residency is one of the PSI requirements. ${ }^{7}$ There was not nursing home resident in our Hospital Information System. As this was a retrospective study, it was not possible to confirm the patient's address. However, there are not many nursing homes in Qinhuangdao. Thirdly, this is a single-center study, and therefore, the results cannot be generalizable to other hospitals or regions in the country or other countries. Consequently, the result should be validated in other populations.

In summary, our data suggest that CURB-65 and PSI class are correlated with in-hospital mortality of CAP in patients with and without T2DM. However, compared with non-diabetic patients, the predictive performance of CURB-65 and PSI class decreased in patients with T2DM. A prediction model for evaluating the CAP severity in the T2DM population should be developed by future studies.

\section{Ethics and Consent Statements}

This was a retrospective study. Data were extracted from the Hospital Information System. We covered patient data confidentiality. Personal information of the patients, such as name and telephone number, were not extracted. Informed consent was waived by the ethics committee of the First Hospital of Qinhuangdao, and this study complied with the Declaration of Helsinki.

\section{Funding}

We have no relevant funding to disclose.

\section{Disclosure}

The authors report no conflicts of interest in this work.

\section{References}

1. Jain S, Self WH, Wunderink RG, et al; Team CES. Communityacquired pneumonia requiring hospitalization among U.S. adults. $N$ Engl J Med. 2015;373(5):415-427. doi:10.1056/NEJMoa1500245

2. Partouche H, Lepoutre A, Vaure CBD, Poisson T, Toubiana L, Gilberg S. Incidence of all-cause adult community-acquired pneumonia in primary care settings in France. Med Mal Infect. 2018;48:389-395. doi:10.1016/j.medmal.2018.02.012

3. Lopardo GD, Fridman D, Raimondo E, et al. Incidence rate of community-acquired pneumonia in adults: a population-based prospective active surveillance study in three cities in South America. BMJ Open. 2018;8(4):e019439. doi:10.1136/bmjopen-2017-019439

4. Takaki M, Nakama T, Ishida $M$, et al. High incidence of community-acquired pneumonia among rapidly aging population in Japan: a prospective hospital-based surveillance. Jpn J Infect Dis. 2014;67(4):269-275. doi:10.7883/yoken.67.269

5. Heo JY, Seo YB, Choi WS, et al. Incidence and case fatality rates of community-acquired pneumonia and pneumococcal diseases among Korean adults: catchment population-based analysis. PLoS One. 2018;13(3):e0194598. doi:10.1371/journal.pone.0194598

6. Lim WS, van der Eerden MM, Laing R, et al. Defining community acquired pneumonia severity on presentation to hospital: an international derivation and validation study. Thorax. 2003;58:377-382. doi:10.1136/thorax.58.5.377

7. Fine MJ, Auble TE, Yealy DM, et al. A prediction rule to identify low-risk patients with community-acquired pneumonia. $N$ Engl J Med. 1997;336(4):243-250. doi:10.1056/NEJM199701233360402

8. Cao B, Huang Y, She D-Y, et al. Diagnosis and treatment of community-acquired pneumonia in adults: 2016 clinical practice guidelines by the Chinese Thoracic Society, Chinese Medical Association. Clin Respir J. 2018;12(4):1320-1360. doi:10.1111/ crj.12674

9. Metlay JP, Waterer GW, Long AC, et al. Diagnosis and treatment of adults with community-acquired pneumonia. An official clinical practice guideline of the American Thoracic Society and Infectious Diseases Society of America. Am J Respir Crit Care Med. 2019;200:e45-e67. doi:10.1164/rccm.201908-1581ST

10. Lim WS, Baudouin SV, George RC, et al; Pneumonia Guidelines Committee of the BTSSoCC. BTS guidelines for the management of community acquired pneumonia in adults: update 2009. Thorax. 2009;64(Suppl 3):iii1-iii55. doi:10.1136/thx.2009.121434

11. Boyles TH, Brink A, Calligaro GL, et al; South African Thoracic S and Federation of Infectious Diseases Societies of Southern A. South African guideline for the management of community-acquired pneumonia in adults. J Thorac Dis. 2017;9(6):1469-1502. doi:10.21037/ jtd.2017.05.31

12. International Diabetes Federation. IDF Diabetes Atlas teB. Belgium; 2019. Available from: http://www.diabetesatlas.org. Accessed March $16,2021$. 
13. Sandler M. Is the lung a "target organ" in diabetes mellitus? Arch Intern Med. 1990;150(7):1385-1388. doi:10.1001/archinte.1990.00 390190051006

14. Koziel H, Koziel MJ. Pulmonary complications of diabetes mellitus: pneumonia. Infect Dis Clin North Am. 1995;9(1):65-96. doi:10.1016/ S0891-5520(20)30641-3

15. Ma CM, Liu Q, Li ML, et al. The effects of type 2 diabetes and postoperative pneumonia on the mortality in inpatients with surgery. Diabetes Metab Syndr Obes. 2019;12:2507-2513. doi:10.2147/ DMSO.S232039

16. Baker EH, Baines DL. Airway glucose homeostasis: a new target in the prevention and treatment of pulmonary infection. Chest. 2018;153 (2):507-514. doi:10.1016/j.chest.2017.05.031

17. Huang H, Guo Q, Li L, et al. Effect of type 2 diabetes mellitus on pulmonary function. Exp Clin Endocrinol Diabetes. 2014;122 (06):322-326. doi:10.1055/s-0034-1372579

18. Torres A, Blasi F, Dartois N, Akova M. Which individuals are at increased risk of pneumococcal disease and why? Impact of COPD, asthma, smoking, diabetes, and/or chronic heart disease on community-acquired pneumonia and invasive pneumococcal disease: table 1. Thorax. 2015;70(10):984-989. doi:10.1136/thoraxjnl-2015206780

19. Koskela HO, Salonen PH, Romppanen J, Niskanen L. Long-term mortality after community-acquired pneumonia-impacts of diabetes and newly discovered hyperglycaemia: a prospective, observational cohort study. BMJ Open. 2014;4:e005715. doi:10.1136/bmjopen2014-005715

20. Martins M, Boavida JM, Raposo JF, et al. Diabetes hinders community-acquired pneumonia outcomes in hospitalized patients. BMJ Open Diabetes Res Care. 2016;4(1):e000181. doi:10.1136/ bmjdrc-2015-000181

21. Konomura K, Nagai H, Akazawa M. Economic burden of community-acquired pneumonia among elderly patients: a Japanese perspective. Pneumonia (Nathan). 2017;9(1):19. doi:10.1186/s41479017-0042-1

22. Zhou H, Guo S, Lan T, Ma S, Zhang F, Zhao Z. Risk stratification and prediction value of procalcitonin and clinical severity scores for community-acquired pneumonia in ED. Am J Emerg Med. 2018;36 (12):2155-2160. doi:10.1016/j.ajem.2018.03.050

23. Man SY, Lee N, Ip M, et al. Prospective comparison of three predictive rules for assessing severity of community-acquired pneumonia in Hong Kong. Thorax. 2007;62(4):348-353. doi:10.1136/thx.20 06.069740
24. Ahn JH, Choi EY. Expanded A-DROP score: a new scoring system for the prediction of mortality in hospitalized patients with community-acquired pneumonia. Sci Rep. 2018;8(1):14588. doi:10. 1038/s41598-018-32750-2

25. Ito A, Ishida T, Tokumasu H, Yamazaki A, Washio Y. Evaluation of pneumonia severity scoring systems in nursing and healthcare-associated pneumonia for predicting prognosis: a prospective, cohort study. J Infect Chemother. 2020;26(4):372-378. doi:10.1016/j.jiac. 2019.11.001

26. Swets JA. Measuring the accuracy of diagnostic systems. Science. 1988;240(4857):1285-1293. doi:10.1126/science.3287615

27. Aujesky D, Auble TE, Yealy DM, et al. Prospective comparison of three validated prediction rules for prognosis in community-acquired pneumonia. Am J Med. 2005;118(4):384-392. doi:10.1016/j. amjmed.2005.01.006

28. Zhang ZX, Yong Y, Tan WC, Shen L, Ng HS, Fong KY. Prognostic factors for mortality due to pneumonia among adults from different age groups in Singapore and mortality predictions based on PSI and CURB-65. Singapore Med J. 2018;59(4):190-198. doi:10.11622/ smedj.2017079

29. Loke YK, Kwok CS, Niruban A, Myint PK. Value of severity scales in predicting mortality from community-acquired pneumonia: systematic review and meta-analysis. Thorax. 2010;65(10):884-890. doi:10.1136/thx.2009.134072

30. Espinoza R, Silva J, Bergmann A, et al. Factors associated with mortality in severe community-acquired pneumonia: a multicenter cohort study. J Crit Care. 2019;50:82-86. doi:10.1016/j.jcrc.2018.11.024

31. Thomas MC, Cooper ME, Zimmet P. Changing epidemiology of type 2 diabetes mellitus and associated chronic kidney disease. Nat Rev Nephrol. 2016;12(2):73-81. doi:10.1038/nrneph.2015.173

32. Lyu Y, Luo Y, Li C, et al. Regional differences in the prevalence of coronary heart disease and stroke in patients with type 2 diabetes in China. J Clin Endocrinol Metab. 2018;103(9):3319-3330. doi:10. 1210/jc.2018-00422

33. Chen H-F, Ho C-A, Li C-Y. Risk of heart failure in a population with type 2 diabetes versus a population without diabetes with and without coronary heart disease. Diabetes Obes Metab. 2019;21(1):112-119. doi:10.1111/dom.13493

34. Shi SJ, Li H, Liu M, et al. Mortality prediction to hospitalized patients with influenza pneumonia: $\mathrm{PO} 2 / \mathrm{FiO} 2$ combined lymphocyte count is the answer. Clin Respir J. 2017;11:352-360. doi:10.1111/ crj. 12346

\section{Publish your work in this journal}

Diabetes, Metabolic Syndrome and Obesity: Targets and Therapy is an international, peer-reviewed open-access journal committed to the rapid publication of the latest laboratory and clinical findings in the fields of diabetes, metabolic syndrome and obesity research. Original research, review, case reports, hypothesis formation, expert opinion and commentaries are all considered for publication. The manuscript management system is completely online and includes a very quick and fair peer-review system, which is all easy to use. Visit http://www.dovepress.com/testimonials.php to read real quotes from published authors. 